\title{
The core populations and co-occurrence patterns of prokaryotic communities in household biogas digesters
}

\author{
Junpeng Rui ${ }^{1,2 \dagger}$, Jiabao $\mathrm{Li}^{1,2 \dagger}$, Shiheng Zhang ${ }^{1,2}$, Xuefeng Yan ${ }^{1,2}$, Yuanpeng Wang ${ }^{3}$ and Xiangzhen Li $\mathrm{i}^{1,2^{*}}$
}

\begin{abstract}
Background: Household biogas digesters are widely used to harvest energy in rural areas of developing countries. Understanding core prokaryotic communities, their co-occurrence patterns, and their relationships to environmental factors is important to manage these small-scale anaerobic digestion systems effectively. In this study, 43 household biogas digesters were collected across eight provinces in China. Prokaryotic communities were investigated using 454 pyrosequencing of $16 \mathrm{~S}$ rRNA genes.
\end{abstract}

Results: Fourteen core genera and ten core OTUs were identified in household biogas digesters. They were mainly affiliated with the phylum Firmicutes, Synergistetes, Actinobacteria, Chloroflexi, and Spirochaetes. Core prokaryotic genera were mainly composed of Clostridium, Clostridium XI, Syntrophomonas, Cloacibacillus, Sedimentibacter, and Turicibacter. Prokaryotic communities in the 43 samples were clearly divided into two clusters. Cluster I was dominated by Clostridium, while Cluster II was dominated by members of Spirochaetes, Bacteroidales, Clostridia, and abundant syntrophs and methanogens. $\mathrm{NH}_{4}{ }^{+}-\mathrm{N}$ and $\mathrm{COD}$ contributed significantly to the assembly of the prokaryotic community in Cluster I, while $\mathrm{NH}_{4}{ }^{+}-\mathrm{N}, \mathrm{pH}$, and phosphate contributed significantly to Cluster II. Correlation-based network analysis showed that the prokaryotic communities in the biogas digesters were dominated by some functional modules. Cluster I was dominated by acetotrophic methanogenic modules and the Clostridium-driven primary fermentation module, while the network of Cluster II was dominated by hydrogenotrophic and acetogenic methanogenesis modules and multi-group-driven (Spirochaetes, Bacteroidales, and Clostridia) primary fermentation modules. The network of Cluster II was more complex and functionally redundant.

Conclusions: Prokaryotic communities identified in the household biogas digesters varied significantly and were affected by environmental factors, such as $\mathrm{NH}_{4}{ }^{+}-\mathrm{N}, \mathrm{pH}$, and COD. However, core prokaryotic communities existed, and most of them were also dominant populations. Cosmopolitan OTUs tended to co-occur. Prokaryotic communities in biogas digesters were well organized by some functional modules. The modular structure of the prokaryotic community, which has functional redundancy, enhances the resistance against environmental stress and maintains digestion efficiency in the anaerobic digestion process.

Keywords: Household biogas digesters, Prokaryotic community, Co-occurrence pattern, Manure digestion, Methanogenesis

\footnotetext{
*Correspondence: lixz@cib.ac.cn

${ }^{\dagger} J u n p e n g$ Rui and Jiabao Li contributed equally to this work

${ }^{2}$ Environmental Microbiology Key Laboratory of Sichuan Province,

Chengdu Institute of Biology, Chinese Academy of Sciences,

Chengdu 610041, China

Full list of author information is available at the end of the article
} 


\section{Background}

Anaerobic digestion is an effective process for converting organic waste, e.g., animal manure and agricultural or food waste, into biogas containing 50-70 \% methane [1, 2]. Generally speaking, digestion consists of four steps: substrate hydrolysis, acidogenesis, acetogenesis, and methanogenesis. The stable and efficient digestion process relies on multiple syntrophic relationships among a community of microbes, including hydrolyzing and fermenting bacteria, acidogenic and acetogenic bacteria, and methanogenic archaea [3, 4]. However, microbial populations in anaerobic manure digesters can be highly variable, even with the digestion of a common core substrate [5]. A deep analysis of the structure and variations of bioreactor microbial communities may potentially reveal their important assembly mechanisms.

Many factors affect the prokaryotic community structure in biogas digesters, including digester design, substrates, and operational conditions [1, 6, 7]. Compared to the large-scale digesters, household biogas digesters are usually small in size that most digesters have volume of less than $10 \mathrm{~m}^{3}$. Geographic difference is likely more important to influence anaerobic digestion process in household biogas digesters. For example, temperature is not controlled during the operation; therefore, the digestion process is affected by the seasonal variation of local climate. Mixed raw materials are usually used depending on their local availability, e.g., manures from livestock, humans, and grass residues. Substrate types and quality are often recognized as the primary driving factors shaping microbial communities in anaerobic biogas digesters [8]. As a digester is constantly re-inoculated by multiple substrates, variations in substrate quantity and quality may lead to different microbiomes. Further, microbiomes in the digesters reflect not only the variation of manure quality, but also differences in the digestive tracts of rumen and non-rumen animals. Swine manure is most often used for household biogas digestion in China. It usually contains high ammonium nitrogen $\left(\mathrm{NH}_{4}{ }^{+}-\mathrm{N}\right)$ due to the high protein content [3]. High $\mathrm{NH}_{4}{ }^{+}-\mathrm{N}$ is an inhibitor of methanogenesis, especially acetotrophic methanogens [9]. Therefore, the concentration of $\mathrm{NH}_{4}{ }^{+}-\mathrm{N}$ may be a crucial factor affecting prokaryotic community structure in the household biogas digester.

A core OTU is usually defined as being present in most samples $[10,11]$. Huse et al. reported that more OTUs will be detected but the differences are minor if using the definition of $90 \%$ prevalence, compared to $95 \%$. The core microorganisms in this study are defined as those common to most digesters (90\% prevalence), while specific microorganisms exist only in a few or in one digester. The variations in both core and specific populations are related to changes in function (i.e., digestion efficiency) and environmental conditions (i.e., operating conditions). Core microorganisms may have a stronger ability to resist perturbation, while specific microorganisms respond rapidly to some changing conditions. Core and specific microorganisms have been identified, based on seven multiple types of digestion systems, using the clone library method [11]. However, the information is limited by the low throughput clone library method and the small number of digester samples. Moreover, core and specific microbial populations can be better identified by using a high throughput sequencing technique and a larger number of samples from biogas digesters.

The anaerobic methanogenic system is a representative model with a well-organized, closely interacting bacterial and archaeal community. Co-occurrence of prokaryotic populations in the system reflects their similar niche adaptation of the co-occurring species, or interspecies interactions, either by competition or by cooperation. In the anaerobic digestion system, nearly all acidogenic microorganisms also participate in hydrolysis, such as members of Clostridium, Ruminococcus, and Bacteroidetes [3]. Acetogenesis could be carried out by at least two groups of bacteria: homoacetogens and syntrophs. Acetogenic syntrophs, e.g., the butyrate oxidizer Syntrophomonas [12], and the benzoate oxidizer Syntrophus [13], can metabolize syntrophically with hydrogenotrophic methanogens. Through the syntrophic metabolism, $\mathrm{H}_{2}$ partial pressure is maintained at a very low level to keep anaerobic oxidation of organic matter energetically [4]. Homoacetogens could exergonically produce acetate, competing for substrates with primary fermenters, secondary fermenters, and hydrogenotrophic methanogens [14]. These interactions are also characterized by a co-occurrence network. The correlation-based co-occurrence network analysis can produce microbial functional modules, which enable us to reveal the interactions between different functional groups and environmental factors in various complex systems [15-19].

Household biogas digesters are widely used to harvest energy in rural China and other developing countries [20]. However, according to the literature review, there are few reports using a pyrosequencing technique to compare bacterial communities between various household biogas digesters operated at different geographic locations. The co-occurrence patterns of prokaryotic communities in the household biogas digesters were not revealed. In this study, we collected sludge samples from 43 household biogas digesters across eight provinces of China, and analyzed the variations and co-occurrence networks of prokaryotic communities based on $16 \mathrm{~S}$ rRNA amplicon pyrosequencing data. The aims were to investigate (1) variations of the prokaryotic community structure, (2) core prokaryotic populations, and (3) the 
co-occurrence networks of prokaryotic communities in household biogas digesters.

\section{Results}

Overall prokaryotic community structure and diversity The prokaryotic communities in 43 household biogas sludge samples were separated into two clusters based on UniFrac distances (PerMANOVA $p<0.001$ ) (Fig. 1). The prokaryotic communities were clustered independently on substrate types (Additional file 1: Figure S1), but related to different locations. Cluster I contained 16 samples, mainly from Pengzhou, Deyang, Jitian, Gejiu, and Lanzhou. Cluster II contained 27 samples mainly from the remaining 10 rural areas. The prokaryotic diversity indices based on the number of OTUs (operational taxonomic units), Chao1 richness, and Shannon's and Simpson's diversity indices, revealed that the prokaryotic diversity of Cluster I was significantly lower than that of Cluster II $(p<0.001)$ (Additional file 1: Figure S2, Additional file 2: Table S1).

The results of principal coordinate analysis (PCoA) showed that the community structures of Cluster I were strongly affected by $\mathrm{NH}_{4}{ }^{+}-\mathrm{N}$, while those of Cluster II were strongly affected by $\mathrm{NH}_{4}{ }^{+}-\mathrm{N}, \mathrm{COD}$ (chemical oxygen demand), and $\mathrm{pH}$ (Fig. 2). Variance partitioning analysis (VPA) was performed to quantify the relative contributions of different environmental variables to changes in the prokaryotic community structure (Additional file 2: Table S2). It showed that COD and $\mathrm{NH}_{4}{ }^{+}$$\mathrm{N}$ were the primary measured environmental factors to affect community structure in Cluster I, explaining 14.8 and $13.6 \%$ of total observed variation, respectively $(p<0.05) . \mathrm{NH}_{4}{ }^{+}-\mathrm{N}$ and $\mathrm{pH}$ explained 18.9 and $14.4 \%$ of total observed variation in Cluster II, respectively, including $9.0 \%$ shared between them $(p<0.01)$. Therefore, $\mathrm{NH}_{4}{ }^{+}-\mathrm{N}$ was the primary environmental factor that influenced community structure in both clusters.

\section{Core prokaryotic populations in biogas sludge}

The 1641 OTUs were detected in these 43 samples based on $97 \%$ identity of $16 \mathrm{~S}$ rRNA gene sequences. The 961 OTUs were shared between Cluster I and II. A total of 61 OTUs $(0.45 \%$ of 1641 OTUs in relative abundance) were detected only in Cluster I, mainly affiliated with Clostridiales. The 619 OTUs (12.31 \% of 1641 OTUs in relative abundance) were detected only in Cluster II, mainly affiliated with Bacteroidetes and Spirochaetes. Generally, the OTUs related to Clostridium, Clostridium XI, Turicibacter, Ruminococcaceae, and Anaerolinaceae were more abundant in Cluster I, while those affiliated with Bacteroidales, Sphaerochaeta, Candidatus Cloacamonas, Porphyromonadaceae, and Methanosaeta were more abundant in Cluster II.
OTUs distributed in $>90 \%$ of the 43 digesters were defined as core OTUs in this study. Results showed that there were 10 core OTUs, mainly affiliated with Firmicutes, such as Clostridium, Clostridium XI, Syntrophomonas, and Turicibacter (Table 1). Members of Cloacibacillus and Anaerolinaceae were also included. Generally, most of the core OTUs were also dominant OTUs with a relative abundance of $>1 \%$, and the sum proportion of them was 45.1 and $16.1 \%$ in Cluster I and II, respectively.

OTUs distributed in $>90 \%$ of samples in each cluster were defined as sub-core OTUs excluding core OTUs. Fourteen sub-core OTUs were identified in Cluster I. They were mainly affiliated with Firmicutes (such as Clostridium, Trichococcus and Lachnospiraceae), Actinobacteria (Cloacibacillus, Leucobacter), and the aerobic Acinetobacter. These 14 OTUs were also presented in many Cluster II samples, but they were less abundant than those in Cluster I (2.3 vs. 6.4 \%) (Additional file 2: Table S3).

Fourteen sub-core OTUs were also identified in Cluster II. They were mainly affiliated with Bacteroidetes (such as Bacteroidales and Porphyromonadaceae) and Spirochaetes (such as Sphaerochaeta, Candidatus Cloacamonas, and Treponema) (Additional file 2: Table S3). The amount of these 14 OTUs was much lower in Cluster I than in Cluster II (1.1 vs. $9.0 \%$ in total), and some of them were not observed in Cluster I.

The definitions of core genera and sub-core genera were similar to those of core OTUs and sub-core OTUs. The 14 core genera were identified, and they were affiliated with Firmicutes, Synergistetes, Actinobacteria, and Spirochaetes. Among them, six core genera contained core OTUs (Table 1). Three sub-core genera in Cluster I and seven in Cluster II (Additional file 2: Table S3) were also identified. The communities of Cluster I mainly consisted of core genera (60.3\% in total), while those of Cluster II mainly consisted of core (31.0\%) and sub-core genera $(10.7 \%)$, indicating that prokaryotic communities were more diverse in Cluster II than in Cluster I.

At the phylum level, Firmicutes were most abundant in both Cluster I and II. Compared to Cluster II, Cluster I digesters had more abundant Firmicutes (75.2 vs. $33.1 \%$ ) and Chloroflexi (7.6 vs. $2.7 \%$ ), and less abundant Bacteroidetes (2.5 vs. $25.8 \%)$, Spirochaetes (0.5 vs. $15.8 \%)$, Euryarchaeota (1.0 vs. $4.2 \%)$, and Tenericutes (0.6 vs. $1.8 \%)(p<0.01$, Fig. 3a, Additional file 2: Table S4).

At the genus level, Clostridium was most abundant in both Cluster I and II. Compared to Cluster II, Cluster I digesters had more abundant Clostridium (34.4 vs. $10.6 \%)$, Clostridium XI (10.4 vs $2.1 \%)$, Turicibacter $(4.5$ vs. $1.6 \%$ ), and Tissierella (1.7 vs. $0.5 \%$ ), and less Sphaerochaeta (0.07 vs. $7.1 \%$ ), Candidatus Cloacamonas (0.4 vs. 


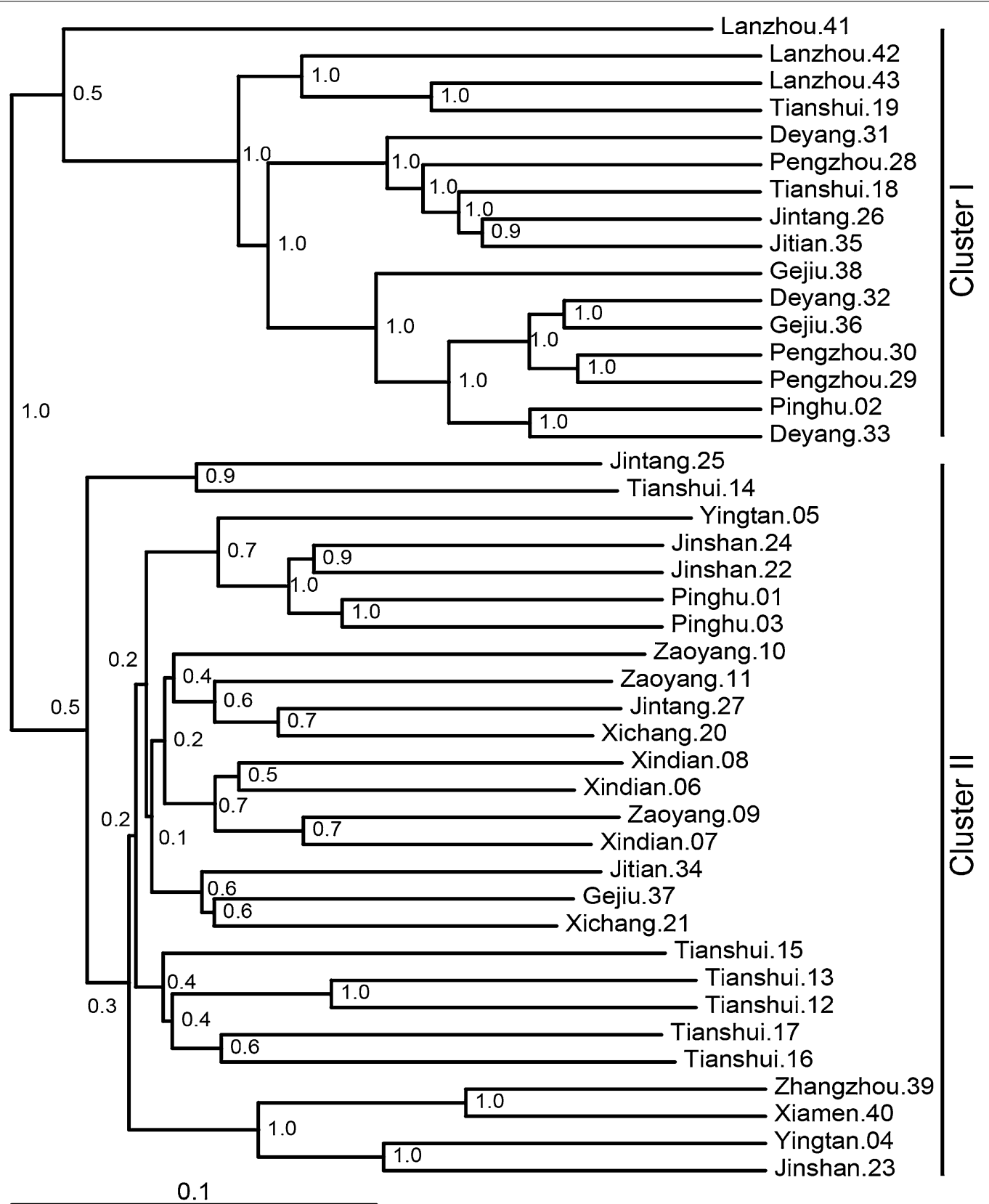

Fig. 1 Jackknife sample cluster analysis of prokaryotic communities based on weighted UniFrac distances. The internal nodes represent values of Jackknife support

$5.3 \%)$, and Treponema (0.04 vs. $1.4 \%)(p<0.01$, Fig. 3b, Additional file 2: Table S4). The relative abundances of Cloacibacillus (about $4 \%$ ) and Syntrophomonas (about $2 \%)$ were similar in both clusters.

Methanogens were more abundant in Cluster II than in Cluster I (3.9 vs. $1.0 \%$ in total reads, $p<0.01$ ). Compared to Cluster I, Cluster II contained more Methanosaeta (1.61 vs. $0.22 \%, p<0.05)$, Methanoculleus $(0.16$ vs. $0.008 \%, p<0.05)$, and Methanospirillum (0.14 vs.
$0.003 \%, p<0.01$ ) (Fig. 3c). Besides, Methanosarcina, Methanocorpusculum, and Methanogenium were also abundant in several samples. In general, acetotrophic (Methanosaeta, Methanosarcina) and hydrogenotrophic methanogens (Methanocorpusculum, Methanogenium, Methanoculleus, Methanospirillum, Methanobrevibacter, etc.) accounted for 46 and $54 \%$ of all methanogens in both clusters, respectively, without a significant difference. 


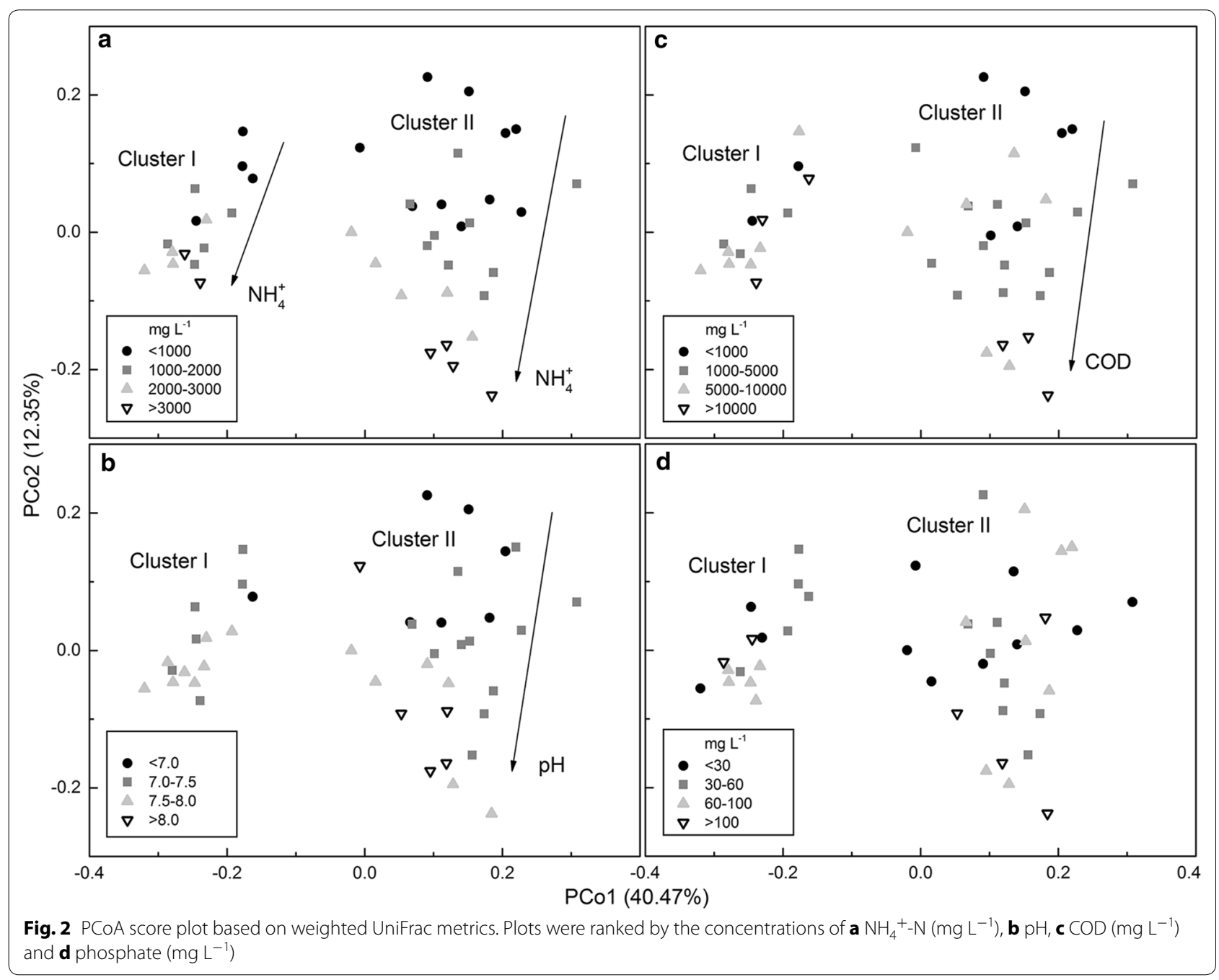

Table 1 Core genera and core OTUs and their average relative abundances in household biogas digesters

\begin{tabular}{|c|c|c|c|c|c|c|c|}
\hline \multirow[t]{2}{*}{ Core genera/OTUs } & \multicolumn{3}{|c|}{ Relative abundance (\%) } & \multirow[t]{2}{*}{ Core genera/OTUs } & \multicolumn{3}{|c|}{ Relative abundance (\%) } \\
\hline & Cluster I & Cluster II & All samples & & Cluster I & Cluster II & All samples \\
\hline Phylum Firmicutes & & & & Phylum Firmicutes & & & \\
\hline Clostridium & $34.37 \pm 4.26$ & $10.56 \pm 1.29$ & $19.42 \pm 2.49$ & Anaerovorax & $0.72 \pm 0.14$ & $0.57 \pm 0.09$ & $0.62 \pm 0.08$ \\
\hline OTU1 & $16.39 \pm 2.63$ & $5.27 \pm 0.90$ & $9.41 \pm 1.38$ & Ruminococcus & $0.45 \pm 0.13$ & $0.66 \pm 0.14$ & $0.58 \pm 0.10$ \\
\hline OTU1200 & $3.27 \pm 0.62$ & $0.59 \pm 0.12$ & $1.59 \pm 0.31$ & Oscillospira & $0.09 \pm 0.02$ & $0.32 \pm 0.10$ & $0.23 \pm 0.06$ \\
\hline OTU24 & $1.29 \pm 0.28$ & $0.51 \pm 0.14$ & $0.80 \pm 0.15$ & Phylum Synergistetes & & & \\
\hline Clostridium XI & $10.36 \pm 1.84$ & $2.09 \pm 0.28$ & $5.17 \pm 0.92$ & Cloacibacillus & $3.90 \pm 1.74$ & $4.05 \pm 1.13$ & $3.99 \pm 0.93$ \\
\hline OTU2 & $5.22 \pm 1.17$ & $1.34 \pm 0.22$ & $2.78 \pm 0.53$ & OTU5 & $3.38 \pm 1.58$ & $3.31 \pm 1.07$ & $3.34 \pm 0.87$ \\
\hline OTU3 & $4.62 \pm 0.95$ & $0.70 \pm 0.12$ & $2.16 \pm 0.46$ & Aminobacterium & $0.52 \pm 0.14$ & $0.85 \pm 0.22$ & $0.73 \pm 0.15$ \\
\hline Turicibacter & $4.53 \pm 0.85$ & $1.57 \pm 0.24$ & $2.67 \pm 0.41$ & Phylum Actinobacteria & & & \\
\hline OTU4 & $4.53 \pm 0.85$ & $1.57 \pm 0.24$ & $2.67 \pm 0.41$ & Corynebacterium & $0.34 \pm 0.16$ & $0.69 \pm 0.20$ & $0.56 \pm 0.14$ \\
\hline Syntrophomonas & $2.01 \pm 0.66$ & $2.19 \pm 0.38$ & $2.12 \pm 0.33$ & Leucobacter & $0.42 \pm 0.14$ & $0.09 \pm 0.02$ & $0.21 \pm 0.06$ \\
\hline OTU9 & $1.63 \pm 0.62$ & $1.08 \pm 0.31$ & $1.28 \pm 0.30$ & Phylum Spirochaetes & & & \\
\hline Sedimentibacter & $0.44 \pm 0.12$ & $1.50 \pm 0.35$ & $1.10 \pm 0.23$ & Candidatus Cloacamonas & $0.39 \pm 0.19$ & $5.35 \pm 1.02$ & $3.50 \pm 0.74$ \\
\hline OTU19 & $0.32 \pm 0.08$ & $1.07 \pm 0.26$ & $0.79 \pm 0.17$ & Phylum Chloroflexi & & & \\
\hline Tissierella & $1.73 \pm 0.48$ & $0.54 \pm 0.15$ & $0.98 \pm 0.21$ & OTU10 (Anaerolinaceae) & $4.41 \pm 1.44$ & $0.67 \pm 0.15$ & $2.06 \pm 0.59$ \\
\hline
\end{tabular}




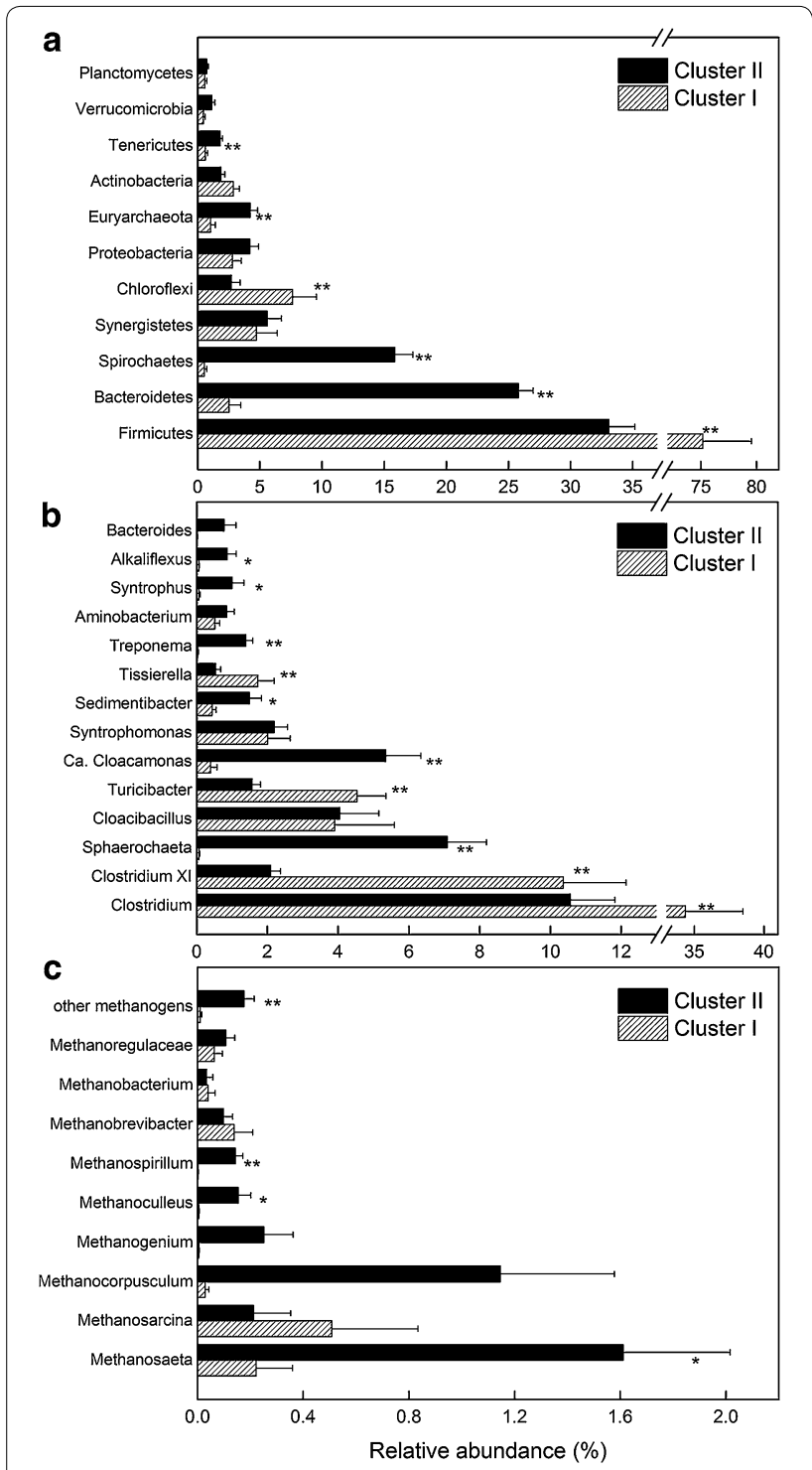

Fig. 3 Taxonomic compositions of microbial communities in Cluster I and II. Relative abundances (\% of total reads) of $16 \mathrm{~S}$ rRNA gene a at the phylum level of prokaryote, $\mathbf{b}$ at the genus level of bacteria, and c at the genus/family level of methanogens. ** Significant at $p<0.01$ *significant at $p<0.05$

\section{Relationships of prokaryotic communities with environmental factors}

Pearson's correlation analysis indicated that the relative abundance of phylum Firmicutes was significantly correlated to phosphate concentration in Cluster I, while it was significantly correlated to $\mathrm{pH}$ in Cluster II (Additional file 2: Table S5). Euryarchaeota (e.g. Methanosaeta) and Syntrophus were negatively correlated with $\mathrm{NH}_{4}{ }^{+}-\mathrm{N}$, indicating that they were sensitive to $\mathrm{NH}_{4}{ }^{+}-\mathrm{N}$ (Additional file 1: Figure S3). However, $\mathrm{NH}_{4}{ }^{+}-\mathrm{N}$ was positively correlated to Spirochaetes and Tenericutes in Cluster II $(p<0.01)$.
Generally, more genera were significantly correlated to COD and $\mathrm{NH}_{4}{ }^{+}-\mathrm{N}$ in Cluster I, while more were significantly correlated to $\mathrm{pH}, \mathrm{NH}_{4}{ }^{+}-\mathrm{N}$, and phosphate in Cluster II (Additional file 2: Table S5). Sphaerochaeta showed a significant positive correlation with $\mathrm{NH}_{4}{ }^{+}-\mathrm{N}, \mathrm{COD}$, phosphate, and $\mathrm{pH}$ in Cluster II. In Cluster II, the genus Clostridium showed positive correlations, while Syntrophus showed negative correlations to $\mathrm{pH}$ and $\mathrm{NH}_{4}{ }^{+}-\mathrm{N}$.

The dominant acetotrophic methanogens (genus Methanosaeta) and hydrogenotrophic methanogens (especially Methanoregulaceae) were significantly and negatively correlated with both $\mathrm{NH}_{4}{ }^{+}-\mathrm{N}$ and $\mathrm{pH}$, while Methanocorpusculum was only negatively correlated with $\mathrm{pH}(p<0.05$, Additional file 2: Table S6). In contrast to other methanogens, Methanoculleus were positively correlated with both $\mathrm{NH}_{4}{ }^{+}-\mathrm{N}$ and $\mathrm{pH}(p>0.05)$. COD was negatively correlated with Methanosaeta and Methanoregulaceae, while positively correlated with Methanobrevibacter $(p<0.05)$. These results indicated that different methanogens were susceptible to different environmental factors.

\section{Network analysis of cosmopolitan OTUs}

Cosmopolitan OTUs were defined as OTUs that occurred in more than half of the samples in the sample group. Cosmopolitan OTUs were identified in Cluster I, II, and in all samples. Nonrandom co-occurrence patterns were detected by the $\mathrm{C}$-score test, with the observed C-scores $(6.78,24.29$, and 65.30, respectively) being higher than the mean values $(6.65,23.56$, and 63.42 respectively, $p<0.0001)$ expected under the null model, indicating that these cosmopolitans tended to co-occur more often than expected by chance.

Three correlation-based networks, named C1, C2, and AS, were constructed with these cosmopolitan OTUs for Cluster I, Cluster II, and all samples, respectively (Fig. 4, Additional file 1: Figure S4). Prokaryotic communities in Cluster II digesters showed different topological properties of co-occurring networks from those in Cluster I digesters (Additional file 2: Table S7). The network sizes were similar in AS and C1 (110 and 103 nodes respectively), but were much smaller than C2 (206 nodes). The total abundance of OTUs that occurred in these networks was $60.4,73.0$, and $65.8 \%$, respectively, indicating that most microorganisms in the sludge samples were affiliated with these cosmopolitan OTUs. Values of modularity, average clustering coefficient, and average path length in these empirical networks were higher than those in random networks, suggesting that the empirical networks had "small world" modularity and hierarchy properties [17, 21].

An important function of each module can be inferred based on the prokaryotic composition, PICRUSt 
prediction, and their known physiological functions $[3,22]$. Cluster I contained eight modules, in which the function of five modules could be predicted confidently. 93 nodes (OTUs) belonged to the module C1M0, 1, 2, 4, and 6 (Table 2, Additional file 2: Table S8), mainly affiliated with Firmicutes (57.9\%), Chloroflexi (6.3\%), and Spirochaetes $(4.2 \%)$. The ammonium-sensitive methanogen Methanosaeta was in the module C1M1, while Methanosarcina was in the high $\mathrm{NH}_{4}{ }^{+}-\mathrm{N}$ module C1M2 (positive correlation with $\mathrm{NH}_{4}{ }^{+}-\mathrm{N}$ ) (Table 3). Large modules C1M1 and C1M2 were predicted to be similar in their function, most likely conducting fermentation mainly with acetotrophic methanogens. C1M4 was also a high- $\mathrm{NH}_{4}{ }^{+}-\mathrm{N}$ module, dominated by Clostridium for fermentation. The small module C1M6 included aerobic or facultative anaerobic Proteobacteria, e.g., Sphingomonas, Methylobacteriaceae, and Acinetobacter, which were likely involved in organic substrate degradation and oxygen consumption for the maintenance of anoxic environment. C1M4 had a positive relationship to C1M1, C1M2, and $\mathrm{C} 1 \mathrm{M} 6$, reflecting their cooperative nature. The negative relationship between $\mathrm{C} 1 \mathrm{M} 1$ and $\mathrm{C} 1 \mathrm{M} 2$ reflected a certain competition (Additional file 2: Table S9).

In the 10-module network $\mathrm{C} 2,198$ nodes belonged to module C2M 0, 1, 2, 4, 5, 6, and 8 (Additional file 2: Table S10), which were composed mainly of Firmicutes (22.4\%), Bacteroidetes (19.1\%), and Spirochaetes $(13.6 \%)$. Among them, 7 functional modules were identified in $\mathrm{C} 2$, including three methanogenic fermentation modules. C2M2 and C2M6 were hydrogenotrophic methanogenic modules, with Methanocorpusculum and Methanogenium as the key methanogen, respectively. C2M5 was an acetotrophic methanogenic module, with Methanosaeta as the key methanogen. C2M1 and C2M8 were primary fermentation modules, including abundant Spirochaetes, Bacteroidetes, and Clostridia. These modules were likely regulated by $\mathrm{NH}_{4}{ }^{+}-\mathrm{N}$. Based on the relationships between its members and $\mathrm{NH}_{4}{ }^{+}-\mathrm{N}$, it is inferred that $\mathrm{C} 2 \mathrm{M} 1$ preferred high $\mathrm{NH}_{4}{ }^{+}-\mathrm{N}$, while $\mathrm{C} 2 \mathrm{M} 2$, 5 , and 8 preferred low $\mathrm{NH}_{4}{ }^{+}-\mathrm{N}$.

$\mathrm{C} 1$ and $\mathrm{C} 2$ shared 53 nodes distributed in almost all modules. They were mainly affiliated with Clostridia and Anaerolinaceae (Additional file 2: Tables S8, S11). The remaining 53 nodes in $\mathrm{C} 1$ were mainly related to Clostridia. However, most of nodes in C1M6 belonged to aerobic Proteobacteria, such as Methylobacterium and Sphingomonas. The remaining 153 nodes in $\mathrm{C} 2$ were mainly affiliated with Spirochaetes and Bacteroidales, and they also included nodes belonging to hydrogenotrophic methanogens (Methanocorpusculum and Methanogenium) and syntrophs (Syntrophus and Syntrophomonas).
In the 11-module network AS, 93 nodes belonged to module AM1, 4, 5, 7, and 8 (Additional file 2: Table S11). These nodes were mainly affiliated with Firmicutes (relative abundance of $31.8 \%$ in total OTUs), Bacteroidetes (9.8 \%), and Spirochaetes (7.5 \%). Hydrogenotrophic methanogens Methanocorpusculum and Methanoculleus were in AM1, and acetogenic Methanosaeta was in AM7. They mainly co-occurred with Bacteroidetes and Spirochaetes (Table 2). Members of AM4 and AM5 were mainly affiliated with Clostridia, especially the genus Clostridium. The network AS was a combination of C1 and $\mathrm{C} 2$, sharing 105 nodes with them (Additional file 1: Figure S5). Nodes in AM1, 7, and 8 were mainly shared with those in $\mathrm{C} 2$, while those in AM4 and 5 were mainly shared with both $\mathrm{C} 1$ and $\mathrm{C} 2$ (Fig. 4b).

Totally, 388, 330, and 771 pairs of nodes were positively correlated in AS, C1, and C2, respectively (Spearman's $\rho>0.6, p<0.01$, Fig. 4), while only 46, 135, and 108 pairs of nodes were negatively correlated, respectively (Spearman's $\rho<-0.6, p<0.01$, not shown as edges in Fig. 4). Negative associations usually existed between Clostridium and Bacteroides/Syntrophus in AS, and Clostridium and Coriobacteriales/Facklamia/Cloacibacillus/Anaerolinaceae in $\mathrm{C} 1$, probably due to the high abundances of Clostridium in many samples (tradeoff or competition interactions). However, negative associations were more complex in $\mathrm{C} 2$ since the amount of Clostridium was much less in these samples. Negative associations usually existed between Clostridium/Tissierella/Bacteroidales and Syntrophus/Treponema, Sphaerochaeta, and Syntrophus/Bacteroidales in $\mathrm{C} 2$.

OTU23 (Methanocorpusculum), OTU142 (Methanoculleus), OTU14 (Methanosaeta) were three nodes belonging to methanogens in AS, with 20,7 , and 7 cooccurrents, respectively (Fig. 4). Their co-occurrents were mainly affiliated to Bacteroidetes and Spirochaetes, which were likely involved in hydrolysis and acidogenesis, and the production of precursors for methanogenesis. OTU14 also co-occurred with acetogenic syntrophs, e.g., Syntrophomonas and Syntrophus. Therefore, these cooccurrence relationships may reflect a food chain cascade or syntrophic interactions in the anaerobic digestion.

The partial Mantel test showed that $\mathrm{NH}_{4}{ }^{+}-\mathrm{N}$ was significantly related to many modules in these three networks, such as AM5, 7 and 8, C1M2 and 4, C2M1, 2, 5, and 6 (Table 3). Modules significantly related to $\mathrm{pH}$ or COD were almost related to $\mathrm{NH}_{4}{ }^{+}-\mathrm{N}$ as well. It was likely that nodes often shared among modules have the same ammonium preference in different networks. Therefore, $\mathrm{NH}_{4}{ }^{+}-\mathrm{N}$ may be an important environmental factor in influencing microbial modularity. 


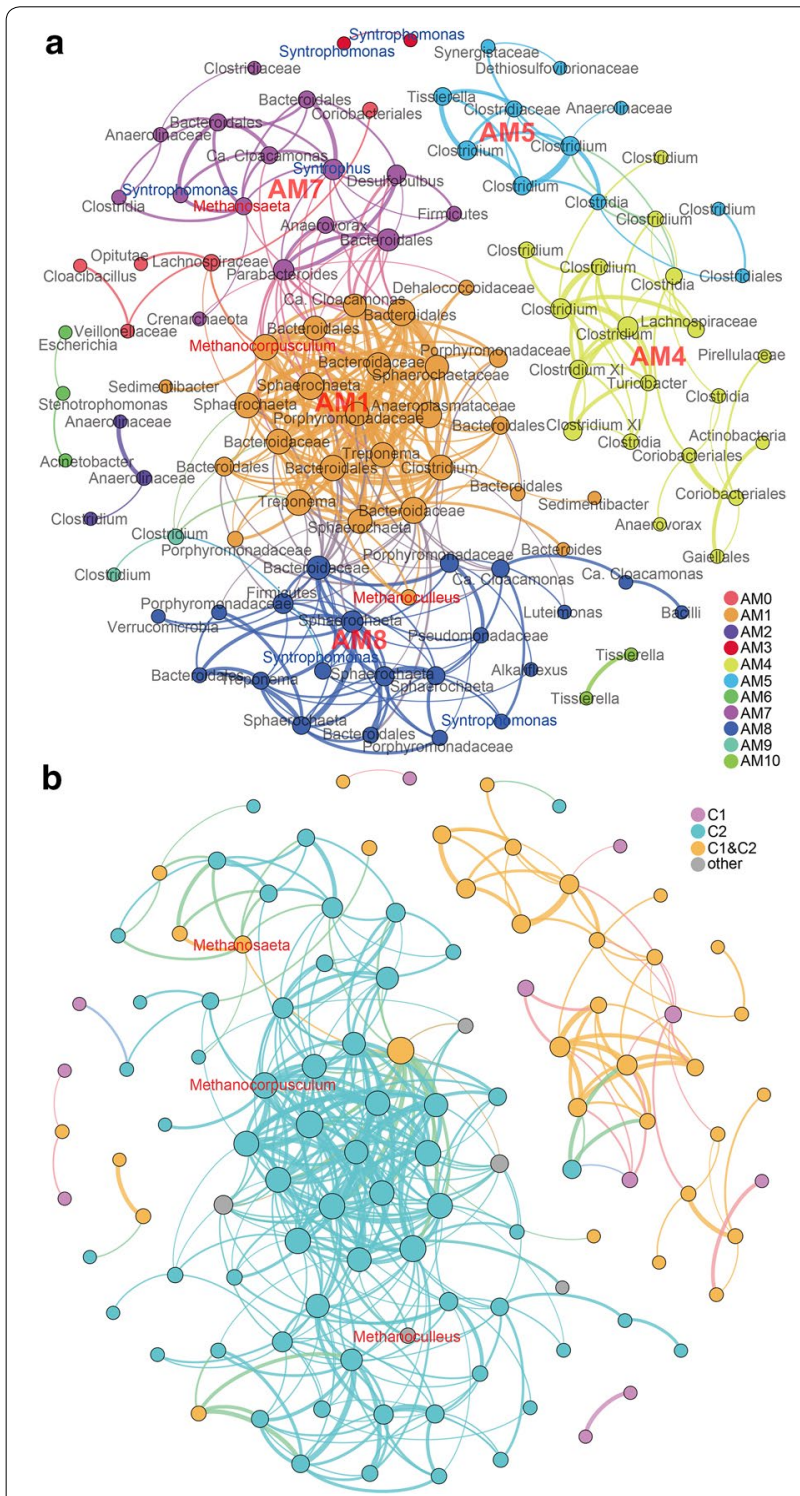

Fig. 4 Networks of co-occurring prokaryotic OTUs in all sludge samples based on correlation analysis. Nodes were colored by a modularity class with labeled genera names, and $\mathbf{b}$ occurrence in networks of Cluster I (C1) and Cluster II (C2). A connection stands for a strong (Spearman's $\rho>0.6)$ and significant $(p<0.01)$ correlation. For each panel, the size of each node is proportional to the number of connections (degree); the thickness of each connection between two nodes (edge) is proportional to the value of Spearman's correlation coefficients, ranging from 0.60 to 0.93 . Other: OTUs did not occur in networks of Cluster I or II

\section{Discussion}

Core prokaryotic communities in the biogas digesters

In this study, 14 core genera were identified, mainly affiliated with the phylum Firmicutes (9 genera), such as Clostridium, Clostridium XI, Syntrophomonas, Sedimentibacter, and Turicibacter. The others were affiliated with the phyla Synergistetes (Cloacibacillus and Aminobacterium), Actinobacteria, and Spirochaetes (Candidatus Cloacamonas). Ten core OTUs were identified, mainly affiliated with Firmicutes, such as Clostridium, Clostridium XI, Syntrophomonas and Turicibacter, Cloacibacillus, and Anaerolinaceae. Generally, most of the core OTUs were also dominant OTUs in biogas digesters, indicating their importance in biogas fermentation, regardless of the treatment process and geographic locations.

Core genera or OTUs identified in this study are also widely detected in various anaerobic digestion systems $[11,23]$. Phylogeny-based empirical relationships can yield powerful correlations between community structure and function as observed in previous studies [24]. Core populations identified in this study have been recognized to play important roles in hydrolysis, fermentation, and syntrophic metabolism. The Genus Clostridium participates in both hydrolysis and acidogenesis, and it is especially dominant in the first two digestion phases. The Clostridium members decompose various substrates, such as starch, cellulose, amino acids, and fatty acids [3]. Members of Clostridium and Bacteroidetes are able to hydrolyze proteins to amino acids with proteases, and degrade amino acids to fatty acids and $\mathrm{NH}_{4}{ }^{+}-\mathrm{N}$ [22]. Clostridium XI was more abundant in Cluster I. It is affiliated with the family Peptostreptococcaceae, which can ferment saccharides, alcohol, and cellulose [25]. Sphaerochaeta was more abundant in Cluster II, which could enhance the degradation of cellulose when grown in coculture with Clostridium thermocellum [26]. Cloacibacillus could ferment amino acids (e.g., mucin in swine intestinal tract), and produce fatty acids [27]. Turicibacter is able to degrade carbohydrates, which is an important member of the gut microbiota [28]. Anaerolinaceae members were more abundant in Cluster I, and they could ferment carbohydrates and produce hydrogen and acetate [29]. The PICRUSt prediction further supported that the genes encoding enzymes involved in polysaccharides hydrolysis existed in some core populations such as Clostridium, Clostridium XI, Sphaerochaeta, Leucobacter, Turicibacter, Bacteroidetes, and Anaerolinaceae. Some of them also include genes encoding proteases, such as Clostridium, Clostridium XI, Sphaerochaeta, Candidatus Cloacamonas, Bacteroidetes, and Anaerolinaceae.

Each full-scale bioenergy system has a unique community structure with an unprecedented level of stability [24]. Core bacterial populations must be key players in maintaining the stability and function of an anaerobic digestion system. Bacterial community structures are resilient, and key populations will be rebounded following disturbances [24]. The aim of this study is to compare the general assembly rules of microbial community across different digesters. Thus, although we only 
Table 2 Taxonomic information of dominant modules in the networks of all samples (AS), Cluster I (C1), and II (C2)

\begin{tabular}{|c|c|c|c|c|}
\hline & $\begin{array}{l}\text { Number } \\
\text { of nodes }\end{array}$ & Module hubs & Methanogens & Abundant phyla/classes \\
\hline (1) AS & 110 & & & \\
\hline AM1 & 27 & $\begin{array}{l}\text { Bacteroidales, Sphaerochaeta, Treponema, } \\
\text { Methanocorpusculum, etc. }\end{array}$ & $\begin{array}{l}\text { Methanocorpusculum, } \\
\text { Methanoculleus }\end{array}$ & Bacteroidetes, Spirochaetes \\
\hline AM4 & 19 & Clostridium, Clostridium XI & & Clostridia \\
\hline AM5 & 11 & Clostridium & & Clostridia \\
\hline AM7 & 15 & $\begin{array}{l}\text { Bacteroidales, Parabacteroides, Desulfobulbus, } \\
\text { Syntrophus }\end{array}$ & Methanosaeta & Bacteroidetes, Spirochaetes, Euryarchaeota \\
\hline AM8 & 21 & Sphaerochaeta, Bacteroidaceae, Porphyromonadaceae & & Bacteroidetes, Spirochaetes, Clostridia \\
\hline (2) C1 & 103 & & & \\
\hline $\mathrm{C} 1 \mathrm{MO}$ & 13 & Coriobacteriales, Leucobacter etc. & & Clostridia, Actinobacteria, Synergistetes \\
\hline C1M1 & 28 & Clostridium, Pirellulaceae, Gaiellales, etc. & Methanosaeta & Clostridia, Chloroflexi, Actinobacteria \\
\hline $\mathrm{C} 1 \mathrm{M} 2$ & 24 & Clostridium, Ruminococcaceae, Erysipelotrichaceae & Methanosarcina & Clostridia \\
\hline $\mathrm{C} 1 \mathrm{M} 4$ & 18 & $\begin{array}{l}\text { Clostridium, Clostridia, Coriobacteriales, } \\
\text { Syntrophomonas }\end{array}$ & & Clostridia \\
\hline C1M6 & 10 & Sphingomonas & & Alphaproteobacteria, Gammaproteobacteria \\
\hline (3) C2 & 206 & & & \\
\hline $\mathrm{C} 2 \mathrm{MO}$ & 9 & & & Clostridia \\
\hline $\mathrm{C} 2 \mathrm{M} 1$ & 36 & $\begin{array}{l}\text { Sphaerochaeta, Bacteroidales, Clostridium, Tissierella, } \\
\text { Treponema, Cloacibacillus, etc. }\end{array}$ & & Spirochaetes, Bacteroidetes, Clostridia \\
\hline $\mathrm{C} 2 \mathrm{M} 2$ & 60 & Coriobacteriales & Methanocorpusculum & Clostridia, Bacteroidetes, Spirochaetes \\
\hline $\mathrm{C} 2 \mathrm{M} 4$ & 9 & & & Clostridia, Sphaerochaeta, Bacilli, Actinobacteria \\
\hline C2M5 & 29 & Bacteroidales, Syntrophus, Syntrophomonas, Treponema & Methanosaeta & $\begin{array}{l}\text { Spirochaetes, Bacteroidetes, Deltaproteobacte- } \\
\text { ria, Clostridia, Chloroflexi, Euryarchaeota }\end{array}$ \\
\hline $\mathrm{C} 2 \mathrm{M} 6$ & 22 & & Methanogenium & Clostridia, Spirochaetes, Bacteroidetes \\
\hline $\mathrm{C} 2 \mathrm{M} 8$ & 33 & & & Clostridia, Spirochaetes, Bacteroidetes \\
\hline
\end{tabular}

collected a one-time sample from each of 43 digesters, it may represent the properties of a bacterial community structure in a specific biogas digester.

\section{Significant variation of prokaryotic community}

In this study, the prokaryotic communities of 43 mesophilic household biogas sludge samples were clearly divided into two clusters based on the UniFrac distances, independent of substrate types (Additional file 1: Figure $\mathrm{S} 1 \mathrm{~B})$ or our measured environmental factors $(\mathrm{pH}, \mathrm{COD}$, $\mathrm{NH}_{4}{ }^{+}-\mathrm{N}$, and phosphate, $p>0.05$ ). This indicated the different key factors in shaping the assemblies of prokaryotic communities. Previous work indicated that the prokaryotic communities of 19 full-scale anaerobic digestion installations were divided into two clusters driven by $\mathrm{NH}_{4}{ }^{+}-\mathrm{N}$ concentration [30]. The low $\mathrm{NH}_{4}{ }^{+}-\mathrm{N}$ cluster was dominant with Bacteroidales, while the high $\mathrm{NH}_{4}{ }^{+}$$\mathrm{N}$ cluster was dominant with Clostridiales. In this study, we observed more aerobic microbial organisms (e.g., Sphingomonas and Pseudomonas) and less abundant methanogens in Cluster I digesters. It might be caused by the recent re-inoculation or other disturbance to the digester system. These results possibly implicated poor performance in Cluster I digesters [31]. Clostridium was the main primary fermenter in Cluster I digesters, while more diversified primary fermenters occurred in Cluster II digesters, including Spirochaetes, Bacteroidetes, and Clostridia. The abundances of these bacteria were highly correlated with those of methanogens (Table 4).

The genus Syntrophus is able to syntrophically oxidize benzoate with hydrogenotrophic methanogens, and produce acetate and $\mathrm{H}_{2}$ [13]. The genus Candidatus Cloacamonas is probably a hydrogen-producing syntroph present in many anaerobic digesters [32]. Both of these genera were significantly higher in Cluster II than in Cluster I, indicating active secondary fermentation in Cluster II digesters. Methanogenic activity appears in the acidogenic phase, but the number of methanogenic archaea obviously increases in the methanogenic phase [3]. Methanogens, especially Methanosaeta, Methanoculleus, and Methanospirillum, were more abundant in Cluster II than in Cluster I $(p<0.05)$, indicating methanogenesis was possibly more active in Cluster II digesters.

\section{Selective inhibition of $\mathrm{NH}_{4}{ }^{+}-\mathrm{N}$ affects prokaryotic community structure}

Many environmental factors influence prokaryotic communities in the biogas digestion system, such as 
Table 3 Spearman's correlation of environmental variables to prokaryotic community structures of dominant modules in networks tested by partial Mantel test (permutations: 9999)

\begin{tabular}{lcccc}
\hline & pH & Phosphate & $\mathbf{N H}_{\mathbf{4}}{ }^{+}-\mathbf{N}$ & COD \\
\hline (1) All samples & $0.223^{* *}$ & 0.046 & $0.264^{* *}$ & $0.162^{*}$ \\
AM1 & 0.056 & 0.111 & 0.021 & $0.138^{*}$ \\
AM4 & -0.011 & -0.026 & 0.012 & 0.006 \\
AM5 & $0.244^{* *}$ & -0.026 & $0.246^{* *}$ & -0.07 \\
AM7 & $0.147^{*}$ & $0.139^{*}$ & $0.347^{* *}$ & $0.323^{* *}$ \\
AM8 & 0.059 & -0.026 & $0.110^{*}$ & 0.044 \\
(2) Cluster I & 0.165 & 0.162 & $0.331^{*}$ & 0.223 \\
C1M0 & $0.263^{*}$ & 0.069 & 0.135 & 0.132 \\
C1M1 & 0.027 & 0.033 & 0.194 & 0.117 \\
C1M2 & 0.084 & -0.065 & $0.401^{* *}$ & $0.394^{* *}$ \\
C1M4 & $0.206^{*}$ & $0.309^{* *}$ & $0.300^{*}$ & 0.177 \\
C1M6 & -0.084 & $0.330^{* *}$ & 0.067 & 0.123 \\
(3) Cluster II & $0.398^{* *}$ & 0.02 & $0.384^{* *}$ & 0.157 \\
C2M0 & 0.061 & -0.051 & 0.03 & -0.083 \\
C2M1 & $0.295^{* *}$ & -0.058 & $0.317^{* *}$ & -0.04 \\
C2M2 & $0.402^{* *}$ & $0.194^{*}$ & $0.487^{* *}$ & $0.203^{*}$ \\
C2M4 & 0.043 & -0.095 & -0.048 & 0.029 \\
C2M5 & $0.167^{*}$ & -0.038 & $0.303^{* *}$ & $0.323^{* *}$ \\
C2M6 & 0.081 & -0.158 & 0.009 & 0.1 \\
C2M8 & 0.164 & 0.004 & $0.184^{*}$ & 0.125 \\
\hline
\end{tabular}

** Significant at $p<0.01,{ }^{*}$ significant at $p<0.05$

When one environmental variable was analyzed by the partial Mantel test, the remaining three environmental variables were controlled

substrates, $\mathrm{pH}$, inoculation, etc. [3, 33]. If one environmental factor predominates the microbial community structure, it may decouple the relationships between community structures and other factors. In this study, it is difficult to collect particular data for household biogas digesters, such as gas production rate, hydraulic retention time, exact substrate compositions, and so forth. Among our measured environmental parameters, the $\mathrm{NH}_{4}{ }^{+}-\mathrm{N}, \mathrm{pH}$, and COD were observed to strongly influence prokaryotic communities in the household digesters. Phosphate, which was positively correlated to $\mathrm{NH}_{4}{ }^{+}-\mathrm{N}(p<0.01)$, had less effect on prokaryotic communities, except for module C1M4 and C1M6 dominant by Clostridium and aerobic Proteobacteria, respectively.

Swine manure as a main substrate used in the Chinese household digesters often contains high $\mathrm{NH}_{4}{ }^{+}-\mathrm{N}$. VPA analysis indicated that $\mathrm{NH}_{4}{ }^{+}-\mathrm{N}$ is an important factor in influencing the prokaryotic community structure in both Cluster I and Cluster II. High $\mathrm{NH}_{4}{ }^{+}-\mathrm{N}$ has an inhibiting effect, and may even be toxic to microbial communities because free ammonia could diffuse passively into cells, causing a proton imbalance and potassium deficiency
[34, 35]. High $\mathrm{NH}_{4}{ }^{+}$ion $\left(>1500 \mathrm{mg} \mathrm{L}^{-1} \mathrm{NH}_{4}{ }^{+}-\mathrm{N}\right)$ also has an inhibiting effect on those species (e.g., methanogens) sensitive to $\mathrm{pH}[3,34]$. The $\mathrm{NH}_{4}{ }^{+}-\mathrm{N}$ concentration of 25 samples were higher than $1500 \mathrm{mg} \mathrm{L}^{-1}$ in this study. Compared to bacteria, methanogenic archaea are more susceptible to $\mathrm{NH}_{4}{ }^{+}-\mathrm{N}$. Moreover, the tolerance of hydrogenotrophic methanogens to ammonium is usually higher than that of acetoclastic Methanosarcina and Methanosaeta [9]. In this study, the relative abundance of Euryarchaeota was negatively correlated with $\mathrm{NH}_{4}{ }^{+}-\mathrm{N}$ in both clusters $(p<0.05)$, while only the most dominant methanogen Methanosaeta was inhibited in Cluster II $(p<0.05)$. This indicated that the keystone populations can be altered by $\mathrm{NH}_{4}{ }^{+}-\mathrm{N}$. The microbial community may select syntrophic acetate oxidation as a significant pathway for forming methane from acetate under high $\mathrm{NH}_{4}{ }^{+}-\mathrm{N}$ concentration [36]. Besides $\mathrm{NH}_{4}{ }^{+}-\mathrm{N}$ concentration, the degree of ammonia inhibition could also be influenced by temperature, $\mathrm{pH}$, volatile fatty acids, and some other ions [34]. It is reported that some ions (e.g., $\mathrm{Na}^{+}, \mathrm{Mg}^{2+}$, and $\mathrm{Ca}^{2+}$ ) could be antagonistic to ammonia inhibition [37]. The adaptations of methanogens to ammonia were also observed [38]. The adaptations might be common for the microbial populations due to diverse substrates and long hydraulic retention time in household biogas digesters.

Core methanogen OTU was not observed, indicating that they are susceptible to environmental changes, e.g., $\mathrm{NH}_{4}{ }^{+}$-N. Besides methanogens, this study observed that some bacteria were also inhibited by $\mathrm{NH}_{4}{ }^{+}-\mathrm{N}$, including Proteobacteria (e.g., Syntrophus) and Planctomycetes in Cluster II. However, some bacteria were positively correlated to $\mathrm{NH}_{4}{ }^{+}$-N, including Clostridium and Sphaerochaeta, Erysipelothrix, and Tissierella. Therefore, the selection of different prokaryotic taxa by $\mathrm{NH}_{4}{ }^{+}-\mathrm{N}$ would shift the community structure through the adjustment of species abundance (species sorting), in which those species genetically better adapted to high $\mathrm{NH}_{4}{ }^{+}-\mathrm{N}$ may outcompete other less well-adapted species.

\section{Co-occurrence patterns of prokaryotic communities}

Co-occurrence network analysis is useful in revealing common system-level properties of prokaryotic communities in the biogas digestion systems. Co-occurrence analysis of microbial taxa from 43 household digesters in this study suggested strong within- and between-domain correlations between different groups of microorganisms within the digesters. It also showed that the prokaryotic communities in biogas digesters are well organized by some functional modules. Significant and positive correlations between members within the modules indicated they may co-occur with mutualism interactions, such as 
Table 4 Cosmopolitan methanogen OTUs and their significant $(p<0.01)$ co-occurrent OTUs in all samples

\begin{tabular}{|c|c|c|c|c|c|}
\hline Cosmopolitan & Co-occurrents & Spearman's $\rho$ & $\begin{array}{l}\text { Co-occurrent } \\
\text { affiliations }\end{array}$ & $\begin{array}{l}\text { Co-occurrent } \\
\text { affiliated phylum }\end{array}$ & $\begin{array}{l}\text { Number of } \\
\text { co-occurring } \\
\text { samples }\end{array}$ \\
\hline \multicolumn{6}{|c|}{ (a) OTU23 (Methanocorpusculum) } \\
\hline & OTU74 & 0.675 & Parabacteroides & Bacteroidetes & 20 \\
\hline & OTU78 & 0.722 & Porphyromonadaceae & Bacteroidetes & 23 \\
\hline & OTU1240 & 0.628 & Bacteroidales & Bacteroidetes & 20 \\
\hline & OTU128 & 0.721 & Sphaerochaetaceae & Spirochaetes & 22 \\
\hline & OTU64 & 0.786 & Bacteroidaceae & Bacteroidetes & 23 \\
\hline & OTU38 & 0.708 & Sphaerochaeta & Spirochaetes & 23 \\
\hline & OTU125 & 0.746 & Anaeroplasmataceae & Tenericutes & 22 \\
\hline & OTU416 & 0.840 & Sphaerochaeta & Spirochaetes & 24 \\
\hline & OTU44 & 0.720 & Candidatus Cloacamonas & Spirochaetes & 23 \\
\hline & OTU169 & 0.826 & Treponema & Spirochaetes & 25 \\
\hline & OTU137 & 0.686 & Bacteroidales & Bacteroidetes & 23 \\
\hline & OTU48 & 0.749 & Bacteroidaceae & Bacteroidetes & 22 \\
\hline & OTU92 & 0.672 & Bacteroidales & Bacteroidetes & 20 \\
\hline & OTU339 & 0.659 & Bacteroidales & Bacteroidetes & 21 \\
\hline & OTU47 & 0.661 & Lachnospiraceae & Firmicutes & 21 \\
\hline & OTU91 & 0.637 & Treponema & Spirochaetes & 23 \\
\hline & OTU303 & 0.651 & Anaerovorax & Firmicutes & 20 \\
\hline & OTU844 & 0.722 & Sedimentibacter & Firmicutes & 24 \\
\hline & OTU8 & 0.604 & Bacteroidales & Bacteroidetes & 26 \\
\hline & OTU72 & 0.859 & Sphaerochaeta & Spirochaetes & 27 \\
\hline \multicolumn{6}{|c|}{ (b) OTU14 (Methanosaeta) } \\
\hline & OTU52 & 0.666 & Porphyromonadaceae & Bacteroidetes & 25 \\
\hline & OTU96 & 0.653 & Syntrophus & Proteobacteria & 17 \\
\hline & OTU749 & 0.657 & Bacteroidales & Bacteroidetes & 23 \\
\hline & OTU12 & 0.689 & Candidatus Cloacamonas & Spirochaetes & 17 \\
\hline & OTU87 & 0.742 & Syntrophomonas & Firmicutes & 28 \\
\hline & OTU66 & 0.724 & Bacteroidales & Bacteroidetes & 23 \\
\hline & OTU119 & 0.703 & Clostridia & Firmicutes & 23 \\
\hline \multicolumn{6}{|c|}{ (c) OTU142 (Methanoculleus) } \\
\hline & OTU327 & 0.610 & Crenarchaeota & Crenarchaeota & 22 \\
\hline & OTU8 & 0.635 & Bacteroidales & Bacteroidetes & 32 \\
\hline & OTU38 & 0.619 & Sphaerochaeta & Spirochaetes & 21 \\
\hline & OTU137 & 0.714 & Bacteroidales & Bacteroidetes & 23 \\
\hline & OTU169 & 0.648 & Treponema & Spirochaetes & 22 \\
\hline & OTU91 & 0.660 & Treponema & Spirochaetes & 22 \\
\hline & OTU454 & 0.628 & Candidatus Cloacamonas & Spirochaetes & 24 \\
\hline
\end{tabular}

an exchange of metabolic intermediates. Methanogenesis is a central metabolic process in the anaerobic biogas digestion. As abundant methanogens in the household biogas digesters, OTUs affiliated to hydrogenotrophic Methanocorpusculum and Methanoculleus and acetoclastic Methanosaeta tended to co-occur with fermentation bacterial Bacteroidetes, Spirochaetes, Tenericutes, and Firmicutes (Table 1). These bacteria participate in hydrolysis and produce intermediates, e.g., $\mathrm{H}_{2} / \mathrm{CO}_{2}$, formate, and acetate [3]. The occurrence of a modularity structure in the prokaryotic community further indicates the occurrence of multiple syntrophic metabolic pathways with functional redundancy of competition or cooperation populations in the biogas digesters. Besides the exchange of metabolic intermediates, multiple syntrophic interactions must be maintained between bacteria and 
methanogens, which consume $\mathrm{H}_{2}$ and maintain a low $\mathrm{H}_{2}$ partial pressure, so that the overall reaction in the system is exergonic [4]. This is further supported by the fact that the positive interactions of multi-group-driven primary fermentation modules with hydrogenotrophic methanogenic fermentation modules were much stronger than those with acetotrophic methanogenic fermentation modules (Additional file 1: Figure S6). The different cooccurrence networks were observed between Cluster I and Cluster II digesters in this study. It was speculated that different co-occurrence networks may influence the stability and performance of biogas digesters.

The assembly of microbial communities is controlled by neutral and deterministic processes [39]. Recent studies indicated that deterministic processes may play a larger role in the process of microbial community assembly in anaerobic digesters [40]. Interspecies interactions and environmental selections are proposed to be two relevant mechanisms of deterministic factors $[41,42]$. The integrative effects of these environmental factors may create niche differentiation, and cause the variations in microbial community structure in various digesters. Further, the results in this study showed that cosmopolitan OTUs tended to co-occur, and microbial communities showed modularity properties in the biogas digesters. These modules and their inferred central functions are highly correlated to some environmental factors, e.g., $\mathrm{NH}_{4}{ }^{+}-\mathrm{N}$, $\mathrm{pH}$, and COD. Thus, the modular structure of microbial interactions may be largely shaped by the deterministic processes.

\section{Conclusions}

The present study showed that 14 genera and 10 OTUs of prokaryotic populations were commonly shared by at least $90 \%$ of all 43 samples. They were mainly affiliated with the phyla Firmicutes, Synergistetes, Actinobacteria, Chloroflexi, and Spirochaetes. Core prokaryotic genera were mainly composed of Clostridium, Clostridium XI, Syntrophomonas, Cloacibacillus, Anaerolinaceae, Sedimentibacter, and Turicibacter. Prokaryotic communities of the 43 samples showed high variations and were clearly separated into 2 clusters with different co-occurrence networks. Cluster I was dominated by Clostridium, while Cluster II was dominated by members of Spirochaetes, Bacteroidales, Clostridia, and abundant syntrophs and methanogens. $\mathrm{NH}_{4}{ }^{+}-\mathrm{N}$ and COD contributed significantly to the assembly of the prokaryotic community in Cluster I, while $\mathrm{NH}_{4}^{+}-\mathrm{N}, \mathrm{pH}$, and phosphate contributed significantly to the community assembly in Cluster II. Correlation-based network analysis showed that the prokaryotic communities of biogas digesters are well organized by some functional modules. These modules and their inferred central functions are highly correlated to some environmental factors, such as $\mathrm{NH}_{4}{ }^{+}-\mathrm{N}, \mathrm{pH}$, and COD. Anaerobic digestion is susceptible to various forms of perturbation because of its delicate balance between the different microbial consortia in the anaerobic digestion process. The modular structure of the prokaryotic community with functional redundancy in the biogas digestion system may provide the system with access to the total functional diversity and environmental specificity available in the community, thus, enhances the resistance against perturbation, and maintains the performance of biogas digesters.

\section{Methods}

Sample description and chemical property measurements Forty-three sludge samples from household biogas digesters were collected in 15 rural areas across eight provinces in China (Additional file 2: Table S12). These digesters, which are also called hydraulic biogas digesters, were typically constructed using brick and concrete in a fixed-dome configuration. All digesters were operated in a temperature range from 18 to $35{ }^{\circ} \mathrm{C}$ without temperature control. The volume of most digesters ranged from 6 to $25 \mathrm{~m}^{3}$. Only one digester had a volume of $55 \mathrm{~m}^{3}$. The feeding substrates varied among individual digesters, including manures from swine, cattle, humans, poultry, and donkeys. Grass residue was used occasionally in some digesters. Usually three bottles of sludge samples from each digester were collected into sterile flasks, transported to the lab under ice, pooled and centrifuged under $8000 \mathrm{rpm}$, and stored at $-20{ }^{\circ} \mathrm{C}$ until the genomic DNA were extracted. Chemical properties of sludge, including $\mathrm{pH}$, chemical oxygen demand, $\mathrm{NH}_{4}{ }^{+}$$\mathrm{N}$, and phosphate were measured as previously described $[43,44]$.

\section{DNA extraction and pyrosequencing}

Genomic DNA was extracted by the method described previously [45]. DNA quality was checked using a NanoDrop Spectrophotometer, subjected to electrophoresis, and visualized in a $0.8 \%$ agarose gel. Extracted DNA was diluted to $10 \mathrm{ng} \mathrm{Hl}^{-1}$ for downstream use. For pyrosequencing, the $16 \mathrm{~S}$ rRNA gene was amplified with universal primers 515F (5'-GTGYCAGCMGCCGCGGTA- $\left.3^{\prime}\right)$ and 909R (5'-CCCCGYCAATTCMTTTRAGT-3'). The detailed PCR conditions were described previously [44]. The barcoded amplicons were pooled with equal molar concentrations of the samples and sequenced using a GS FLX + pyrosequencing system (454 Life Sciences).

\section{Sequencing data analysis}

The raw sequences were sorted based on unique barcodes, trimmed for sequence quality, and clustered at $97 \%$ identity for OTUs with USEARCH v7.0 (http:// 
www.drive5.com/usearch/download.html) using UPARSE pipeline [46]. Chimeras and singletons were removed from clustered sequences with USEARCH. Re-sampling to the same sequence depth (2230 sequences per sample) was performed using daisychopper.pl (http://www. festinalente.me/bioinf/downloads/daisychopper.pl) prior to downstream analysis. Chao1 estimator of richness and Shannon's and Simpson's diversity indices were calculated using QIIME pipeline v1.7.0 (http://qiime.org/ tutorials/tutorial.html) [47]. The phylogenetic affiliation of each sequence was analyzed by an RDP Classifier at a confidence level of $80 \%$ [48]. Gene functions of dominant OTUs were predicted using PICRUSt [49], a tool that predicts the gene function of a microbial community using an existing database of microbial genomes. It is usually used well in predicting the function of microbiome from simple habitats, such as human and animal gut. Recently it is also used to study soil microbiome [50]. To predict the gene function of an OTU, the OTU representative sequence is assigned to a reference sequence in the GreenGenes database at $97 \%$ identity using QIIME. Then, the functional profile of the reference sequence is found in COG and/or KEGG orthology databases using PICRUSt.

The original pyrosequencing data from this study were available at the European Nucleotide Archive by accession no. PRJEB10542 (http://www.ebi.ac.uk/ena/data/ view/PRJEB10542).

\section{Statistical analysis}

Overall structural changes of prokaryotic communities were evaluated by PCoA in Fast UniFrac [51]. The statistical significance among datasets was assessed by PerMANOVA using the weighted PCoA scores in PAST (http://folk.uio.no/ohammer/past/). The partial Mantel test was applied to evaluate the correlations among prokaryotic communities with environmental variables. Variance partitioning analysis (VPA) was performed to quantify the relative contributions of environmental variables based on redundancy analysis (RDA) using the $\mathrm{R}$ package Vegan (http://cran.r-project.org/web/packages/vegan/index.html). One-way-analysis of variance (ANOVA), regression and correlation analysis between prokaryotic abundances and environmental factors were conducted using SPSS 21 software.

\section{Co-occurrence network analysis}

OTUs occurred in more than half of samples were used for network analysis. Non-random co-occurrence patterns of selected OTUs were tested with the checkerboard score $(C$-score) under a null model $[15,52]$. Spearman's rank correlations between selected OTUs were calculated [16]. A valid co-occurrence event was considered to be a robust correlation if the Spearman's correlation coefficient was $\rho>0.6$ with a significance of $p<0.01$ [15]. Correlation networks were constructed with the robust correlations as weighted edges using Gephi software (https://gephi. github.io/). 10,000 Erdös-Réyni random networks with the same number of nodes and edges as the empirical networks were generated using the R package igraph (http:// cran.r-project.org/web/packages/igraph/) [16].

\section{Additional files}

\begin{abstract}
Additional file 1: Figure S1. PCoA score plot based on weighted UniFrac metrics colored by (A) locations, and (B) substrates. P: swine manure; $\mathrm{B}$ : cattle manure; $\mathrm{H}$ : human manure; $\mathrm{C}$ : poultry manure; $\mathrm{E}$ : donkey manure; G: grass. Figure S2. Rarefaction curve of observed OTUs before re-sampling. Figure S3. Relationships between (A) $\mathrm{NH}_{4}{ }^{+}-\mathrm{N}$ concentration and the relative abundance of Euryarchaeota in Cluster II, (B) the relative abundance of Clostridium and that of Euryarchaeota, and (C) the relative abundance of Bacteroidetes and that of Spirochaetes in all samples. Figure S4. Networks of co-occurring prokaryotic OTUs in (A) Cluster I and (B) Cluster II based on correlation analysis. OTUs were colored by modularity class with labeled genera names. A connection stands for a strong (Spearman's $\rho>0.6)$ and significant $(p<0.01)$ correlation. For each panel, the size of each node is proportional to the number of connections (degree); the thickness of each connection between two nodes (edge) is proportional to the value of Spearman's correlation coefficients ranging from 0.60 to 0.95. Ca.: Candidatus. Figure S5. Number of shared nodes (OTUs) among networks AS, C1, and C2. Figure S6. Relationships among functional modules of prokaryotic communities of (A) Cluster I and (B) Cluster II. The shapes of each module represent the main function of the module. The color of each module represents the correlation between the module and $\mathrm{NH}_{4}{ }^{+}-\mathrm{N}$ concentration: black, positive correlation $(p<0.05)$; white, negative correlation ( $p<0.05$ ); grey, no significant correlation. The thickness of each solid line between modules is proportional to the sum of positive Spearman's $\rho$ between them in the networks ( $\mathrm{C} 1$ and $\mathrm{C} 2$ ) ranging from 0.6 to 18.5; a dotted line represents over 10 couples of OTUs with significant negative correlations (Spearman's $\rho<-0.6, p<0.01$ ) between modules.
\end{abstract}

Additional file 2: Table S1. Prokaryotic diversity indices based on $97 \%$ identity of 165 rRNA gene sequences and 2230 reads per sample. Table S2. The relative contributions ( $R$ square value) of each environmental factor to OTUs in all samples, Cluster I and II based on RDA analysis. Table S3. Relative abundances of core genera/OTUs in all 43 samples and sub-core genera/OTUs in Cluster I and II, and their correlation to environmental factors. Table S4. Relative abundances of the abundant phyla (average relative abundance $>0.1 \%$ ) and genera (average relative abundance $>0.05 \%$ ). Table S5. Pearson's correlation of abundant phyla and genera to environmental factors in Cluster I and II. Table S6. Pearson's correlation of abundant methanogens to environmental factors in all samples. Table S7. Topological properties of co-occurring networks AS (43 samples), C1 (27 samples in Cluster I), and C2 (16 samples in Cluster II), generated with Gephi software. Table S8. Node information of 103 cosmopolitan OTUs in the network C1 (Cluster I). Table S9. Positive and negative interactions among modules in network AS, C1, and C2. Table S10. Node information of 206 cosmopolitan OTUs in the network C2 (Cluster II). Table S11. Node information of 110 cosmopolitan OTUs in the network AS (all samples). Table S12. Fermentation conditions and chemical properties in biogas digesters.

\section{Abbreviations}

ANOVA: analysis of variance; COD: chemical oxygen demand; OTU: operational taxonomic unit; PCOA: principal coordinates analysis; PerMANOVA: permutational multivariate analysis of variance; RDA: redundancy analysis; VPA: variance partitioning analysis. 


\section{Authors' contributions}

$J R$ and $J L$ performed the experimental work, data analysis, and writing. $J L$ and YW were involved in the experimental work. JL, SZ and XY participated in sample collections. XL was involved in the experimental design and writing. All authors read and approved the final manuscript.

\section{Author details}

${ }_{1}^{1}$ Key Laboratory of Environmental and Applied Microbiology, Chengdu Institute of Biology, Chinese Academy of Sciences, Chengdu 610041, China.

${ }^{2}$ Environmental Microbiology Key Laboratory of Sichuan Province, Chengdu Institute of Biology, Chinese Academy of Sciences, Chengdu 610041, China.

${ }^{3}$ Department of Chemical and Biochemical Engineering, College of Chemistry and Chemical Engineering, Xiamen University, Fujian 361005, China.

\section{Acknowledgements}

We appreciate many volunteers who helped with sampling. This work was supported by 973 project (No. 2013CB733502), National Key Technology Support Program (2014BAD02B04) and the National Natural Science Foundation of China $(31300447,41371268,41271260)$, CAS database project (XXH125043-18). The authors declare no conflict of interests.

\section{Compliance with ethical guidelines}

\section{Competing interests}

The authors declare that they have no competing interests.

Received: 1 July 2015 Accepted: 9 September 2015

Published online: 25 September 2015

\section{References}

1. Town JR, Links MG, Fonstad TA, Dumonceaux TJ. Molecular characterization of anaerobic digester microbial communities identifies microorganisms that correlate to reactor performance. Bioresour Technol. 2014;151:249-57.

2. Luo G, Angelidaki I. Co-digestion of manure and whey for in situ biogas upgrading by the addition of $\mathrm{H}_{2}$ : process performance and microbial insights. Appl Microbiol Biotechnol. 2013;97(3):1373-81.

3. Deublein D, Steinhauser A. Biogas from waste and renewable resources: an introduction. Weinheim: Wiley-VCH Verlag GmbH \& Co. KGaA; 2008

4. Schink B. Energetics of syntrophic cooperation in methanogenic degradation. Microbiol Mol Biol Rev. 1997;61(2):262-80.

5. St-Pierre B, Wright AD. Comparative metagenomic analysis of bacterial populations in three full-scale mesophilic anaerobic manure digesters. Appl Microbiol Biotechnol. 2014;98(6):2709-17.

6. Lin L, Wan C, Liu X, Lee DJ, Lei Z, Zhang Y, et al. Effect of initial pH on mesophilic hydrolysis and acidification of swine manure. Bioresour Technol. 2013;136:302-8

7. Smith AM, Sharma D, Lappin-Scott H, Burton S, Huber DH. Microbial community structure of a pilot-scale thermophilic anaerobic digester treating poultry litter. Appl Microbiol Biotechnol. 2014;98(5):2321-34.

8. Zhang W, Werner JJ, Agler MT, Angenent LT. Substrate type drives variation in reactor microbiomes of anaerobic digesters. Bioresour Technol. 2014;151:397-401.

9. Karakashev D, Batstone DJ, Angelidaki I. Influence of environmental conditions on methanogenic compositions in anaerobic biogas reactors. Appl Environ Microbiol. 2005;71(1):331-8.

10. Huse SM, Ye YZ, Zhou YJ, Fodor AA. A core human microbiome as viewed through 16S rRNA sequence clusters. PLoS One. 2012;7(6):e34242.

11. Riviere D, Desvignes V, Pelletier E, Chaussonnerie S, Guermazi S, Weissenbach J, et al. Towards the definition of a core of microorganisms involved in anaerobic digestion of sludge. ISME J. 2009;3(6):700-14.

12. Mclnerney MJ, Bryant MP, Hespell RB, Costerton JW. Syntrophomonas wolfei gen. nov. sp. nov., an anaerobic, syntrophic, fatty acid-oxidizing bacterium. Appl Environ Microbiol. 1981;41(4):1029-39.

13. Mountfort DO, Brulla WJ, Krumholz LR, Bryant MP. Syntrophus buswellii gen. nov., sp. nov:: a benzoate catabolizer from methanogenic ecosystems. Int J Syst Bact. 1984;34(2):216-7.
14. Drake H, Küsel K, Matthies C. Acetogenic prokaryotes. In: Dworkin M, Falkow S, Rosenberg E, Schleifer K-H, Stackebrandt E, editors. The prokaryotes. New York: Springer; 2006. p. 354-420.

15. Barberan A, Bates ST, Casamayor EO, Fierer N. Using network analysis to explore co-occurrence patterns in soil microbial communities. ISME J. 2012;6(2):343-51.

16. Ju F, Xia Y, Guo F, Wang Z, Zhang T. Taxonomic relatedness shapes bacterial assembly in activated sludge of globally distributed wastewater treatment plants. Environ Microbiol. 2014;16(8):2421-32.

17. Deng Y, Jiang YH, Yang Y, He Z, Luo F, Zhou J. Molecular ecological network analyses. BMC Bioinform. 2012;13:113.

18. Zhou JZ, Deng Y, Luo F, He ZL, Yang YF. Phylogenetic molecular ecological network of soil microbial communities in response to elevated $\mathrm{CO}_{2}$. $\mathrm{MBio}$. 2011;2(4):e00122-11.

19. Yao M, Rui J, Li J, Dai Y, Bai Y, Heděnec $P$, et al. Rate-specific responses of prokaryotic diversity and structure to nitrogen deposition in the Leymus chinensis steppe. Soil Biol Biochem. 2014;79:81-90.

20. Chen Y, Yang GH, Sweeney S, Feng YZ. Household biogas use in rural China: a study of opportunities and constraints. Renew Sust Energ Rev. 2010;14(1):545-9.

21. Watts DJ, Strogatz SH. Collective dynamics of 'small-world' networks. Nature. 1998;393(6684):440-2.

22. Miyamoto K. Renewable biological systems for alternative sustainable energy production FAO Agricultural Services Bulletin, vol. 128. Rome: Food and Agriculture Organization of the United Nations; 1997.

23. Nelson MC, Morrison M, Yu ZT. A meta-analysis of the microbial diversity observed in anaerobic digesters. Bioresour Technol. 2011;102(4):3730-9.

24. Werner JJ, Knights D, Garcia ML, Scalfone NB, Smith S, Yarasheski K, et al. Bacterial community structures are unique and resilient in full-scale bioenergy systems. Proc Natl Acad Sci USA. 2011;108(10):4158-63.

25. Gaston LW, Stadtman ER. Fermentation of ethylene glycol by Clostridium glycolicum, sp. n. J Bacteriol. 1963;85:356-62.

26. Abt B, Göker M, Scheuner C, Han C, Lu M, Misra M, et al. Genome sequence of the thermophilic fresh-water bacterium Spirochaeta caldaria type strain $(\mathrm{H} 1 \mathrm{~T})$, reclassification of Spirochaeta caldaria, Spirochaeta stenostrepta, and Spirochaeta zuelzerae in the genus Treponema as Treponema caldaria comb. nov., Treponema stenostrepta comb. nov., and Treponema zuelzerae comb. nov., and emendation of the genus Treponema. Stand Genomic Sci. 2013;8(1):88-105.

27. Looft T, Levine UY, Stanton TB. Cloacibacillus porcorum sp. nov., a mucindegrading bacterium from the swine intestinal tract and emended description of the genus Cloacibacillus. Int J Syst Evol Microbiol. 2013;63(Pt 6):1960-6.

28. Bosshard PP, Zbinden R, Altwegg M. Turicibacter sanguinis gen. nov., sp. nov., a novel anaerobic, Gram-positive bacterium. Int J Syst Evol Microbiol. 2002;52(Pt 4):1263-6.

29. Yamada T, Sekiguchi Y, Hanada S, Imachi H, Ohashi A, Harada H, et al. Anaerolinea thermolimosa sp. nov., Levilinea saccharolytica gen. nov., sp. nov. and Leptolinea tardivitalis gen. nov., sp. nov., novel filamentous anaerobes, and description of the new classes Anaerolineae classis nov. and Caldilineae classis nov. in the bacterial phylum Chloroflexi. Int J Syst Evol Microbiol. 2006;56(Pt 6):1331-40.

30. De Vrieze J, Saunders AM, He Y, Fang J, Nielsen PH, Verstraete W, et al. Ammonia and temperature determine potential clustering in the anaerobic digestion microbiome. Water Res. 2015;75:312-23.

31. De Vrieze J, Gildemyn S, Vilchez-Vargas R, Jáuregui R, Pieper D, Verstraete $W$, et al. Inoculum selection is crucial to ensure operational stability in anaerobic digestion. Appl Microbiol Biotechnol. 2015;99(1):189-99.

32. Pelletier E, Kreimeyer A, Bocs S, Rouy Z, Gyapay G, Chouari R, et al. "Candidatus Cloacamonas acidaminovorans": genome sequence reconstruction provides a first glimpse of a new bacterial division. J Bacteriol. 2008;190(7):2572-9.

33. Nabarlatz DA, Arenas-Beltran LP, Herrera-Soraca DM, Nino-Bonilla DA. Biogas production by anaerobic digestion of wastewater from palm oil mill industry. Ct F-Cienc Tecn Fut. 2013;5(2):73-83.

34. Chen Y, Cheng JJ, Creamer KS. Inhibition of anaerobic digestion process: a review. Bioresour Technol. 2008;99(10):4044-64.

35. Sprott GD, Patel GB. Ammonia toxicity in pure cultures of methanogenic bacteria. Syst Appl Microbiol. 1986;7(2-3):358-63.

36. Werner JJ, Garcia ML, Perkins SD, Yarasheski KE, Smith SR, Muegge $\mathrm{BD}$, et al. Microbial community dynamics and stability during an 
ammonia-induced shift to syntrophic acetate oxidation. Appl Environ Microbiol. 2014;80(11):3375-83.

37. Braun R, Huber P, Meyrath J. Ammonia toxicity in liquid piggery manure digestion. Biotechnol Lett. 1981;3(4):159-64.

38. Melbinge NR, Donnello J. Toxic effects of ammonia nitrogen in high-rate digestion. J Water Pollut Con F. 1971;43(8):1658-70.

39. Zhou J, Liu W, Deng Y, Jiang YH, Xue K, He Z, et al. Stochastic assembly leads to alternative communities with distinct functions in a bioreactor microbial community. MBio. 2013;4(2):e00584-12.

40. Vanwonterghem I, Jensen PD, Dennis PG, Hugenholtz P, Rabaey K, Tyson GW. Deterministic processes guide long-term synchronised population dynamics in replicate anaerobic digesters. ISME J. 2014;8(10):2015-28.

41. Stegen JC, Lin X, Konopka AE, Fredrickson JK. Stochastic and deterministic assembly processes in subsurface microbial communities. ISME J. 2012;6(9):1653-64.

42. Zhou J, Deng Y, Zhang P, Xue K, Liang Y, Van Nostrand JD, et al. Stochasticity, succession, and environmental perturbations in a fluidic ecosystem. Proc Natl Acad Sci USA. 2014;111(9):E836-45.

43. Shen L, Hu HY, Ji HF, Cai JY, He N, Li QB, et al. Production of poly(hydroxybutyrate-hydroxyvalerate) from waste organics by the twostage process: focus on the intermediate volatile fatty acids. Bioresour Technol. 2014;166:194-200.

44. Li JB, Rui JP, Pei ZJ, Sun XR, Zhang SH, Yan ZY, et al. Straw- and slurry-associated prokaryotic communities differ during co-fermentation of straw and swine manure. Appl Microbiol Biotechnol. 2014;98(10):4771-80.
45. Rademacher A, Zakrzewski M, Schluter A, Schonberg M, Szczepanowski R, Goesmann A, et al. Characterization of microbial biofilms in a thermophilic biogas system by high-throughput metagenome sequencing. FEMS Microbiol Ecol. 2012;79(3):785-99.

46. Edgar RC. UPARSE: highly accurate OTU sequences from microbial amplicon reads. Nat Methods. 2013;10(10):996-8.

47. Caporaso JG, Kuczynski J, Stombaugh J, Bittinger K, Bushman FD, Costello EK, et al. QIIME allows analysis of high-throughput community sequencing data. Nat Methods. 2010;7(5):335-6.

48. Wang Q, Garrity GM, Tiedje JM, Cole JR. Naïve Bayesian classifier for rapid assignment of rRNA sequences into the new bacterial taxonomy. Appl Environ Microbiol. 2007;73(16):5261-7.

49. Langille MGI, Zaneveld J, Caporaso JG, McDonald D, Knights D, Reyes JA et al. Predictive functional profiling of microbial communities using $16 \mathrm{~S}$ rRNA marker gene sequences. Nat Biotechnol. 2013;31 (9):814-23.

50. Zarraonaindia I, Owens SM, Weisenhorn P, West K, Hampton-Marcell J, Lax $\mathrm{S}$, et al. The soil microbiome influences grapevine-associated microbiota. MBio. 2015;6(2):e02527-14.

51. Lozupone C, Hamady M, Knight R. UniFrac-an online tool for comparing microbial community diversity in a phylogenetic context. BMC Bioinform. 2006; $7: 371$

52. Stone L, Roberts A. The checkerboard score and species distributions. Oecologia. 1990;85(1):74-9.

\section{Submit your next manuscript to BioMed Central and take full advantage of:}

- Convenient online submission

- Thorough peer review

- No space constraints or color figure charges

- Immediate publication on acceptance

- Inclusion in PubMed, CAS, Scopus and Google Scholar

- Research which is freely available for redistribution

Submit your manuscript at 